\title{
Real time calibration for RSS indoor positioning systems
}

\author{
Ana M. Bernardos, José R. Casar, Paula Tarrío \\ Universidad Politécnica de Madrid, Madrid, Spain \\ \{abernardos, jramon, paula\}@grpss.ssr.upm.es
}

\begin{abstract}
Due to the random characteristics of the indoor propagation channel, received signal strength-based localization systems usually need to be manually calibrated once and again to guarantee their best performance. Calibration processes are costly in terms of time and resources, so they should be eliminated or reduced to a minimum. In this direction, this paper presents an optimization algorithm to automatically calibrate a propagation channel model by using a Least Mean Squares technique: RSS samples gathered in a number of reference points (with known positions) are used by a LMS algorithm to calculate those values for the channel model's constants that minimize the error computed by a hyperbolic triangulation positioning algorithm. Preliminary results on simulated and real data show that the localization error in distance is effectively reduced after a number of training samples. The LMS algorithm's simplicity and its low computational and memory costs make it adequate to be used in real systems.
\end{abstract}

Keywords - Indoor positioning, localization, calibration, optimization, LMS.

\section{INTRODLICTION}

The majority of indoor localization systems work with received signal strength (RSS) measurements gathered from different wireless technologies (WiFi. Bluetooth, ZigBee, etc.). The RSS signal random nature makes these systems (using either map-based or channel model based techniques for positioning) need an off-line calibration phase - at least when starting the system for the first time.

The first objective of calibration is to characterize the noisy wireless channel, affected by multipath and fading effects. Secondly, calibration is also needed to detect (and handle) the temporal propagation dynamics originated by unstable environmental conditions (such as humidity), space reorganization (e.g. furniture re-colocation or openclosed doors) and people's movement (temporal flow, human clusters around the mobile target, etc.). For example. [1] analyzes how the average position accuracy of a fingerprint-based system (offering $2.13 \mathrm{~m}$. of accuracy in standard conditions - no-blocking people, close-all-doors and $40 \%$ humidity level) is deteriorated in a $43.7 \%$ when the humidity level increases until $70 \%$, in a $236.6 \%$ if the configuration changes to all-open-doors and in a $85.9 \%$ when people clusters are present. With respect to the last issue, Chan et al. [2] shows the effects of human clustering: a group of people walking around the target user can create strong interferences. which make the positioning accuracy and precision deteriorate. Finally, calibration processes are usually required when new hardware is integrated in a localization system, as the variable characteristics of transceivers chipsets, antennas and packaging of mobile devices commonly degrades the performance of the localization solution [3].

In this paper, a strategy for dynamic calibration of channel model-based localization systems is presented. We assume that the propagation channel adjust to a theoretical lognormal model. which needs to be dynamically adapted to fit the real conditions of operation. The proposed teclinique is based on the existence of several (position-known) reference points, on which it is possible to opportunistically take signal strength measurements (thanks to the interaction with mobile users or to a beacon-organized infrastructure). Afterwards, an algorithm iteratively calculates the values for the constants of the propagation channel model using real-time measurements. The adaptive algorithm works to minimize the error between the estimated position and the real one. As it is iterative - just handling data of the previous temporal instant - and simple in its formulation. the algorithm has minimum computational and memory needs; this means that it can be integrated in real-time localization systems without requiring significant resources and without introducing serious operational delays.

The remainder of the paper is organized as follows. Section II reviews some previous proposals to automate calibration procedures for indoor localization systems. In Section III, our localization scenario is fully described. Section IV gathers the theoretic foundations of the proposed algorithm. The feasibility of the algorithm is shown in Section V through a number of experiments in a real environment. Finally, Section VI concludes the work.

\section{RELATED WORK}

Information about received signal strength is inherent to every wireless communication technology so, when facing the development of localization systems, RSS has been the traditional basis to work with. In brief, RSS indoor positioning systems usually rely on an off-line calibration phase, which aims at characterizing the electromagnetic environment by: 1) calibrating a theoretical propagation model with real RSS measurements or 2) building a RSS fingerprint (or radio 
map) of the localization area (see e.g. [4] for a review on indoor localization systems and techniques).

Calibration processes are costly and inefficient. so a line of research in indoor localization is devoted to propose solutions with zero or limited initial calibration and on-line recalibration.

For example in the calibration phase, Gwon and Jain [5] use inter-anchor RSS measurements to generate. for each anchor, multiple linear functions representing the relationship between RSS and distance. During the online phase, a target client uses the mapping functions of the anchor with strongest RSS $\left(A_{1}\right)$ to calculate its position to the rest of anchors $\left(A_{2} \ldots A_{11}\right)$, and the mapping function for the second anchor $\left(A_{2}\right)$ with strongest RSS to calculate the position to $A_{1}$. A similar strategy is followed by Barsocchi et al. [6]; they propose a calibration strategy by which each anchor in the infrastructure broadcast a beacon that is afterwards used by other anchors to measure the reciprocal RSS. Next the localization server calibrates the propagation model parameters using these data and some information about the space's geometry, adaptively calculating the attenuation factor introduced by the walls.

Lim et al. [7] takes as input the on-line measurements of RSS between (WiFi) anchors, and between a client and its neighboring anchors. to create a mapping between the RSS measurement and the actual geographical distance basing on the tnuncated singular value decomposition teclinique. The algorithm does not rely on a specific propagation model, but on exploiting the fact that the RSS is inversely proportional to the distance to the power of a path loss exponent. Authors claim that the only calibration process this system requires is five minutes of measurements between pairs of anchor nodes.

Apart from stationary emitters (anchors). Krishnan et al. [8] include sniffers in their RF deployment. Sniffers are wireless transmitters that listen for all communications from wireless clients and anchors. Authors use a spline interpolation technique on these data to build a RSS fingerprint. During the on-line localization phase, when at least one sniffer observes a significant deviation on the RSS from any emitter, the floor model is recalculated. Moraes and Nunes [9] also propose a sniffer-based technique to build a propagation map, in which each grid position is associated to a probability distribution. The map is rebuilt every $\mathrm{T}$ seconds or when significant variations in the RSSI occur.

In order to compensate the environmental dynamics, Yin et al. [10] suggest temporally adapting the static RSS fingerprint. RF receivers are distributed over the space as reference points. On the signal strength values received both by the reference points and the mobile target, a multiple regression analysis is used to build a corrected radio map. Information about the location of the reference points is not needed: the system works to learn the predictive relationship of signal-strength values between the reference points and the mobile device.

A multi-fingerprint solution to the problem of environmental dynamics is proposed by Chen et al. [1]. Information from several sensors (passive RFID, humidity sensors, smart cards and short-range Bluetooth) serve to characterize the environmental situation in terms of humidity, open/closed doors and people and to feed an online calibration process which allows building multiple context-aware radio maps. Following, the adaptive localization phase selects the context-aware radio map that best matches the current environmental condition state (calculated from the information coming from environmental sensors).

Zheng et al. [11] propose to automate the calibration process of an RF deployment with a time-based localization teclnology covering selected areas: ultrasound trilateration is used to label RF measurements in the calibration phase. This approach may be feasible when different types of localization granularities are needed, but in most of cases will require a redundant infrastructure

The LANDMARC system is based on deploying passive RFID reference tags to increase the accuracy of their active RFID localization strategy [12]. As described in the next Section, our general approach shares this basic idea, but applies it to the calibration problem: we propose to complete the localization infrastructure with an auxiliary structure of easily deployable and maintainable reference points, with known positions, which will serve to capture real-time RSS data which will train a propagation model by using a Least Mean Square (LMS) algorithm. As far as we know. this optimization technique has not been applied to the integration of on-line RSS measurements in propagation models. and may result in a robust and time-efficient way of performing calibration.

\section{FUNDAMENTALS: LOCALIZATION SCENARIO AND SERVICE REQUIREMENTS}

Let us consider an indoor space covered by a network of anchor nodes (e.g. WiFi or Bluetooth access points. or Zigbee motes). deployed to measure the RSS of mobile targets in order to localize them. Our localization system is based on using a propagation channel model to compute each mobile-anchor node distance and perform hyperbolic triangulation.

The most popular channel model for RSS-based localization is the lognormal model [13]:

$P_{R Y}(d B m)=A-10 \eta \log \frac{d}{d_{0}}+N(0, \sigma)$

where $P_{R X}$ is the received power (at the receiving nodes, respectively), $d$ is the distance between transmitter and receiver, $A$ and $\eta$ are the parameters of the channel model and $N$ is a zero-mean Gaussian random variable with standard deviation $\sigma . A$ depends on the antenna gain. the transmission power and the power loss for a reference distance $d_{0}$ and needs to be experimentally adjusted. The path loss exponent $\eta$ has to be experimentally determined too. For example. in $2.4 \mathrm{GHz}$ IEEE 802.15 .4 propagation. $A$ may range between -50 and -85 , while $\eta$ may be between 1.9 and 3.5 [15].

Using eq. I. given $A$ and $\eta$. the system estimates the distances $d$ from the received $P_{R Y}$ (in practice the RSS). at 
least to three anchor nodes. Next, the target's position is calculated by performing hyperbolic triangulation. The hyperbolic positioning algorithm may be solved with a least squares estimator (detailed formulation is available in e.g. [13]), for which the estimated coordinates $(\hat{x}, \hat{y})$ are obtained by calculating:

$\left[\begin{array}{l}\hat{x} \\ \hat{y}\end{array}\right]=\left(H^{T} H\right)^{-1} H^{T} \tilde{b}$

where:

$$
\begin{gathered}
H=\left[\begin{array}{cc}
2 x_{2} & 2 y_{2} \\
\vdots & \vdots \\
2 x_{N} & 2 y_{N}
\end{array}\right] ; \tilde{b}=\left[\begin{array}{c}
x_{2}^{2}+y_{2}^{2}-\tilde{d}_{2}^{2}+\tilde{d}_{1}^{2} \\
\vdots \\
x_{N}^{2}+y_{N}^{2}-\tilde{d}_{N}^{2}+\tilde{d}_{1}^{2}
\end{array}\right] \\
\tilde{d}_{i}=10^{\frac{A-R S s_{i}}{10 \eta}}
\end{gathered}
$$

being $\left(x_{i}, y_{i}\right)$ the known coordinates of the $i=1 . N$ anchors, $d_{i}$ the distance from the target to each anchor with the origin of coordinates in the anchor node $i=1$, and $R S S_{i}$, the received signal strength to/from each anchor node.

As said before, in practice, both $A$ and $n$ need to be experimentally determined off-line and continually updated or calibrated (slightly biased estimations of $A$ and $\eta$ may result in significant localization errors [13]). In this context our objective is to avoid any off-line experimental determination of $A$ and $\eta 1$ ) to minimize the complexity of the calibration tasks when getting the location system to work for the first time and 2) to adapt the system's performance to real time environmental variations. To do so, we define a number of beacon or reference points located in fixed geographic locations. These reference points, easy to deploy may be situated in waypoints (e.g. doors), attached to static objects (e.g. a printer in an office), or deployed as part of the communications infrastructure (i.e. an anchor node could serve as reference point).

The anchor nodes will measure the RSS coming from these reference points when possible (e.g. when a user with a suitable device is detected on a reference point) and use the algorithm presented in the next section to compute $\mathrm{A}$ and $\eta$ in real time.

Of course, this approach has many practical implementation details that are not directly addressed in this paper. For example, the number of reference points needs to be minimized. even if a technologically hybrid, low-cost and non-intrusive strategy to set them up is feasible. For example, in [12]. one reference tag is needed for each square meter to provide an error distance between one and two meters; this high-density deployment is probably unrealistic in most part of common localization scenarios. Apart from that the physical distribution of the reference points needs to be flexible. as it is not always easy to place new elements in daily-living environments. Additionally, reference points should be easily maintainable and admit dynamic reconfiguration. Assuming that a suitable deployment is feasible (as it is), the optimization algorithm used to calibrate the system is described in the next Section.

\section{THE LEAST MEAN SQUARES (LMS) ADAPTIVE CALIBRATION ALGORITHM}

Least Mean Squares is a well-known gradient-based teclinique to find in a computationally efficient way the values of the parameters of a function of data that approximates or estimates a set of reference values. It is based on approximating the true gradient of the squared error of a function by its instantaneous estimate [14].

In this case, we propose to use it to set up an adaptive filter to minimize the localization error (eq. 5) by recursively adapting the constant parameters of the lognormal propagation model, A and $\eta$.

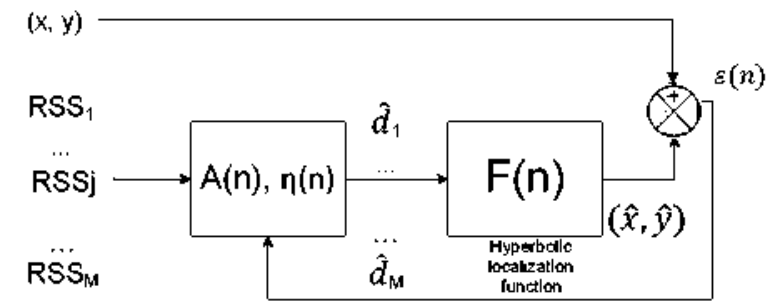

Figure 1. Adaptive filter to minimize the error in location estimation by adapting $A$ and $\eta$.

Figure 1 represents how the adaptive filter works: the LMS algorithm uses the $M$ RSS measurements taken from the calibration/reference points to iteratively calculate the optimal values of $A$ and $\eta$ in each instant (iteration) $n$, to minimize the error between the estimated $(\hat{x}, \hat{y})$ and the (known) real position of the $i$ reference points $\left(x_{i}, y_{i}\right)$. The error to be adaptively minimized is formulated as:

$\varepsilon_{i}(n)=\sqrt{\left(x_{i}(n)-\hat{x}_{i}(n)\right)^{2}+\left(y_{i}(n)-\hat{y}_{j}(n)\right)^{2}}$

In Figure 1, $\mathbf{F}(\mathbf{n})$ represents the hyperbolic localization function (eqs. 1-4) which allows calculating $(\hat{x}, \hat{y})$ with $A(n-l)$ and $\eta(n-1)$; these estimated coordinates will serve to calculate $A(n)$ and $\eta(n)$ as shown in eqs. 6-7.

Assuming that a single channel model is used to characterize the propagation behavior from each anchor node, the Least Mean Square algorithm is formulated as:

$A(n)=A(n-1)-\frac{1}{2} \mu_{A} \frac{\partial E\left[\varepsilon^{2}(n)\right]}{\partial A} \cong A(n-1)-\mu_{A} \varepsilon(n) \frac{\partial \varepsilon(n)}{\partial A}$

$\eta(n) \doteq \eta(n-1)-\mu_{\eta} \varepsilon(n) \frac{\partial \varepsilon(n)}{\partial \eta}$

where $\mu$ s are the filter step sizes that condition and control the speed and stability of convergence (as shown in Section V).

After detailed calculations (basically derivations and simplifications) on the formulas of lyperbolic triangulation (see [13]) the following expressions for $A(n)$ and $\eta(n)$ are obtained:

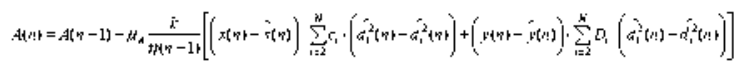

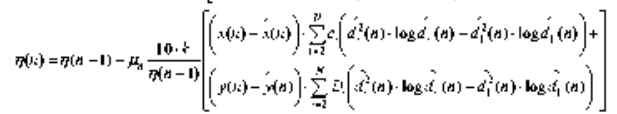

where: 


$$
\begin{aligned}
& k=\frac{-16 \cdot \ln 10}{\operatorname{det} \cdot 10 \cdot \eta(n-1)} \\
& \hat{d}_{i}=10^{\frac{A(n-1)-R \Phi_{i}}{10 \cdot(n-1)}} \\
& c_{i}=x_{i} \cdot\left(y_{2}^{2}+\ldots+y_{N}^{2}\right)-y_{i} \cdot\left(x_{2} \cdot y_{2}+\ldots+x_{N} \cdot y_{N}\right) \\
& D_{i}=y_{1} \cdot\left(x_{2}^{2}+\ldots+x_{N}^{2}\right)-x_{1} \cdot\left(x_{2} \cdot y_{2}+\ldots+x_{N} \cdot y_{N}\right) \\
& \operatorname{det}=\left(4 \cdot x_{2}^{2}+\ldots+4 \cdot x_{N}^{2}\right) \cdot\left(4 \cdot y_{2}^{2}+\ldots+4 \cdot y_{N}^{2}\right)-\left(4 \cdot x_{2} \cdot y_{2}+\ldots+4 \cdot x_{N} \cdot y_{N}\right)^{2}
\end{aligned}
$$

It is important to remark that the recursive values for $A$ and $\eta$ aim at minimizing the distance error; in general, we do not seek convergence to any 'nominal' $A$ and $\eta$ values.

\section{VALIDATION AND RESULTS}

The formulation above has been implemented in Matlab for validation and testing. Following, the LMS algorithm performance is shown by using both simulated and empirical data in a real scenario.

\section{A. Simulation results}

The objective of our simulation analysis has been to test the convergence of the LMS algorithm in a noisy environment. Figure 2 shows our simulation scenario, which is composed by 8 anchor nodes (serving as communications infrastructure) and 20 reference nodes. Actually, this deployment exists in our lab and has been used for empirical validation too (see Section V.B).

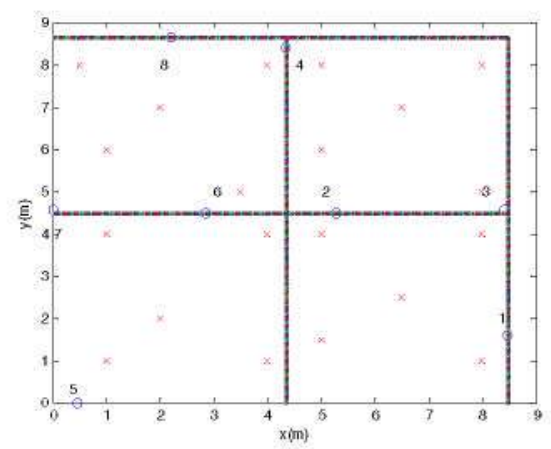

Figure 2. Scenario for simulation: 4 rooms, 8 anchor nodes (o) and 20 reference (beacon) points $(\mathrm{x})$.

The reference points have been randomly chosen, trying to get enough spatial diversity in each room with a moderate number of beacons ( 5 per room, located near the corners and the center), in order to work on a simulated but feasible scenario. In reality, these reference points should be related to objects or waypoints capable of generating 'measurement events'. For example, a door with automatic access control can serve as reference point: when a user is detected to be opening the door (e.g. by processing data from a RFID reader placed in the entrance), the localization infrastructure may trigger a 'measurement event' to request the user's mobile phone to send the (WiFi) RSS received from the nearby access points. In our simulation, 'measurement events' are generated in each reference point: 200 data arrays gathering RSS measurements from every anchor node have been simulated by using eq. 1 , with $\mathrm{P}_{\mathrm{TX} \text {-sim }}=0 \mathrm{dBm}$, $\mathrm{d}_{0 \text {-sim }}=1 \mathrm{~m}, \mathrm{~A}_{\text {sim }}=-60 \mathrm{~dB}, \eta_{\mathrm{sim}}=2.3$ and $\sigma_{\text {sim }}=2.5$ (possible values for a 'standard' indoor propagation model in this geometry, as shown in [15]).

In the first iteration, the hyperbolic localization algorithm (eq. 2) has been then initialized with some given initial values $\left(A_{0}\right.$ and $\left.\eta_{0}\right)$ and fed by a simulated RSS array corresponding to the set of received signal strengths from a randomly chosen reference point to each anchor node. Then, we have obtained the first location estimation and its error - calculated as the mean of the error obtained in each reference point. Afterwards, the obtained values have served as input for eq. 6 , to calculate the values for $A$ and $\eta$ minimizing the distance error. Those values are to be used by the hyperbolic localization algorithm in the next iteration.
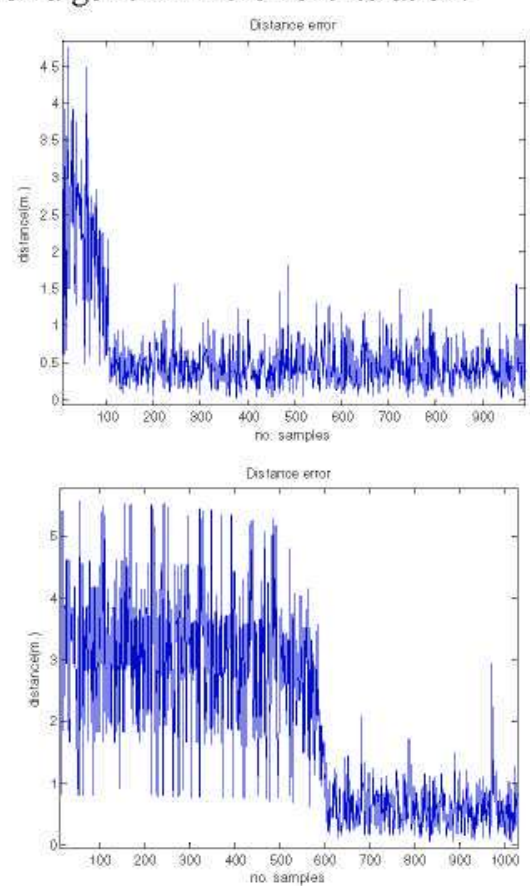

Figure 3. Evolution of the error (m.) in distance using a LMS filter with $\eta_{0}=3, \mu_{A}=0.1, \mu_{\eta}=0.01$ and two different values for $A:$ a) $A_{0}=-65$ dB, b) $A_{0}=-75 \mathrm{~dB}$.

Fig. 3 shows how the LMS algorithm is able to reduce the location/estimation error by adaptively adjusting $\mathrm{A}$ and $\eta$. The case represented in Fig. 3a. starts with a value for $\mathrm{A}_{0}$ just differing $5 \mathrm{dBs}$ from the value used to simulate the RSS measurements $\left(A_{\text {sim }}\right)$. In this case, approximately 100 samples are needed to calibrate the model ( 5 samples per reference point). When the difference between $A_{\text {sim }}$ and $A_{0}$ is $15 \mathrm{dBs}$ (Fig. 3b), around 600 samples are needed to stabilize the error value. In the worst case, when $A_{\text {sim }}$ and $A_{0}$ differ in 25 $\mathrm{dBs}$, approximately 4000 samples are needed (in average, 200 samples per reference point).

The limit for the distance error variance is theoretically imposed by the noise parameter $\sigma$ that has been used to generate the simulated samples (also depends on $\eta$ ).

A significant factor for the LMS algorithm is the filter step sizes $(\mu \mathrm{s})$, which control the pace to convergence but also the stability of the estimation. For our experiments, the step sizes have been empirically chosen among those that where offering a reasonable convergence time (in 
terms of needed number of RSS tuples) while providing a reasonably stable convergence value for $A$ and $\eta$. Although there are other values which may be effectively used, Figure 3 shows how the convergence works for the distance error when the filter step sizes have been set to $\mu_{\mathrm{A}}=0.1, \mu_{\eta}=0.01$. As shown in Figure 4 , higher values of $\mu$ may accelerate the convergence (fewer samples are needed to reach the possible minimum error in distance), but may also provide a less stable convergence.

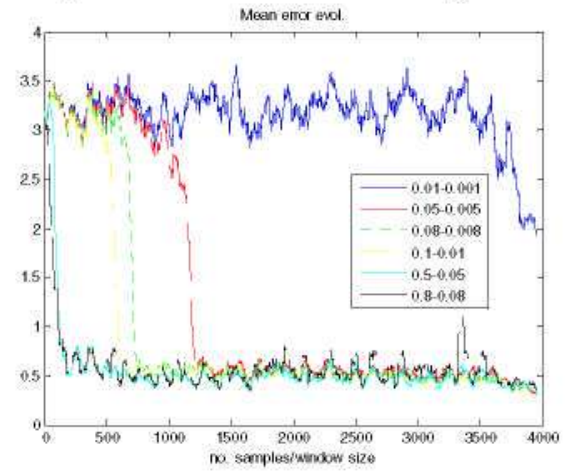

Figure 4. Effects of the value of the LMS filter coefficients $\left\langle\mu_{A}, \mu_{7}\right\rangle$ on the mean error evolution (in m.). LMS initialization: $A_{0}=-75, \eta_{0}=3$. Simulated samples are generated with $A=-60$ and $\eta=2.3$. The mean value is calculated with a sliding window of 50 samples over the error in distance.

Figure 5 shows how $A$ and $\eta$ evolve for several combinations of step sizes. At the beginning, all the curves have a similar behavior towards a convergence value. Afterwards, the convergence stability is affected by the non-linear interactions between the two parameters.
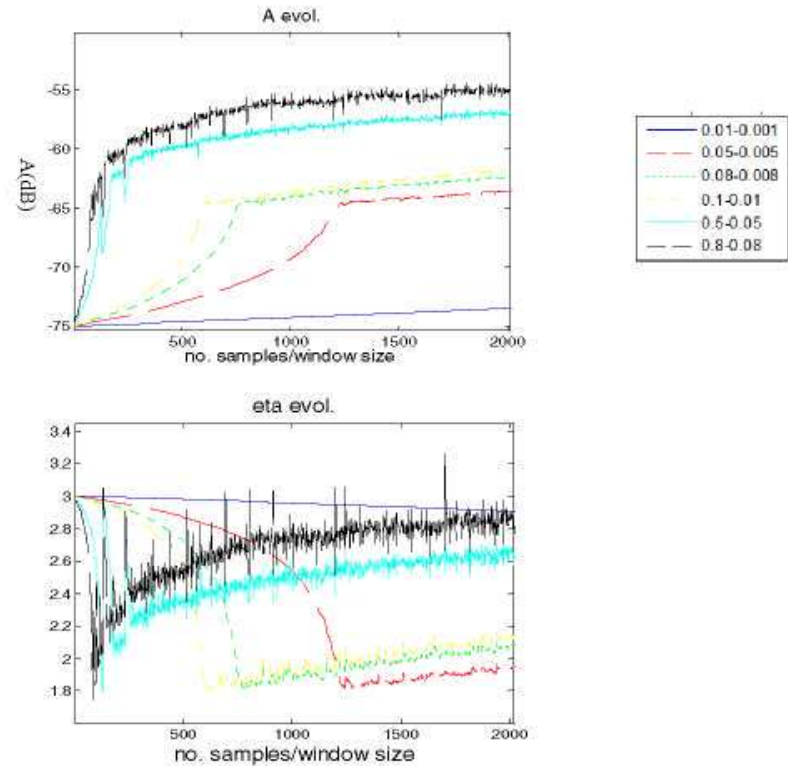

Figure 5. Effects of the value of the LMS filter coefficients $\left(\mu_{A}, \mu_{\eta}\right)$ on the $A$ and $\eta$ iteratively estimated values. LMS initialization: $A_{0}=-75 \mathrm{~dB}$, $\eta_{0}=3$. Simulated samples are generated with $A=-60 \mathrm{~dB}$ and $\eta=2.3 \mathrm{~dB}$. The mean value is calculated with a sliding window of 50 samples over the error in distance.

Figure 6 shows how the algorithm converges when only $A$ is calculated with the LMS, while $\eta$ remains invariable. As $A$ is relevant term in eq. 1, it prevails over $\eta$, assuring convergence (although not to the simulation value) even with biased (non real) values of $\eta$.
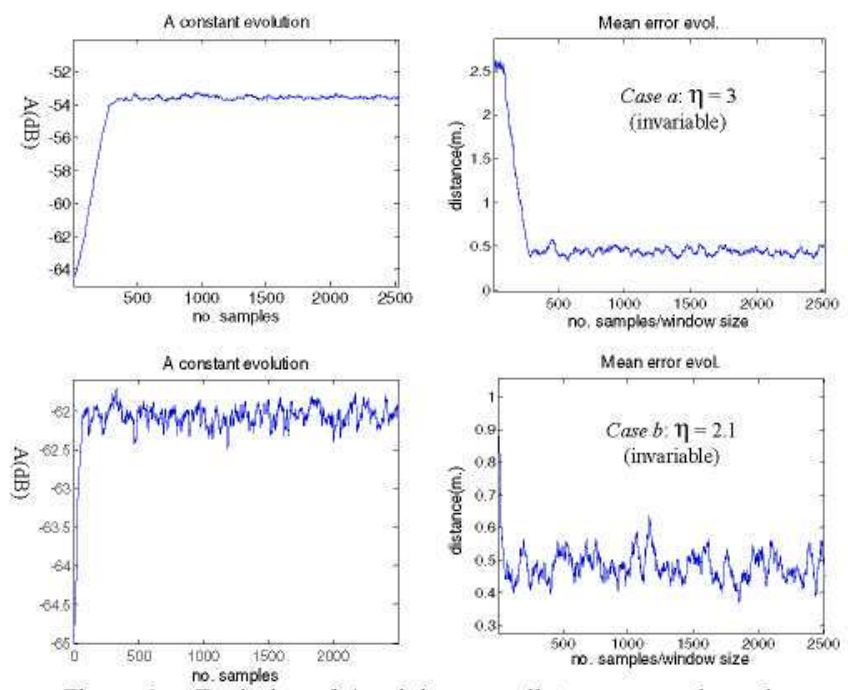

Figure 6. Evolution of $A$ and the mean distance error when $\eta$ is invariable and set to a) 3 or b) $2.1 . A_{0}=-65 \mathrm{~dB}$ and the LMS step sizes are $\mu_{\mathrm{A}}=0.1, \mu_{\eta}=0.01$. Simulated samples have been generated with $\mathrm{A}=$ $60 \mathrm{~dB}$ and $\eta=2.3$. The mean value is calculated with a sliding window of 50 samples over the error in distance.

\section{B. Empirical validation}

The scenario for empirical validation has the same geometry that the simulated one. It is covered by a wireless sensor network working at $2.4 \mathrm{GHz}$, composed by MicaZ motes (an IEEE 802.15.1 compliant hardware platform [16]) as anchor nodes.

With respect to the reference points, 24 beacons have been opportunistically located depending on the furniture and waypoints (doors) of the area (Fig. 7). The distribution seeks to accommodate the 'spatial diversity' and 'reasonable number of reference points' criteria used in the simulated environment, while considering the practical feasibility of the deployment.

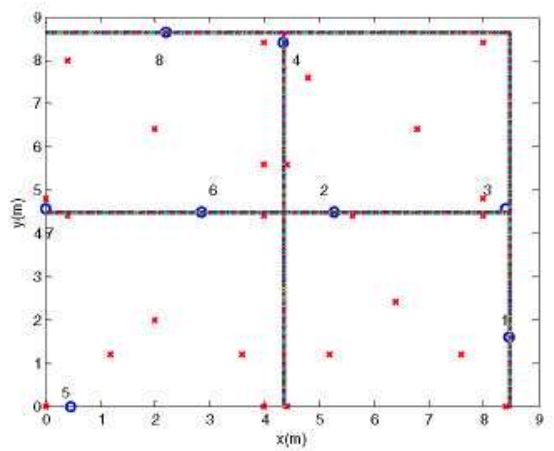

Figure 7. Scenario for empirical validation: 4 rooms, 8 anchor nodes (in blue color) and 24 reference points (in red color). In iteration, a reference point is randomly selected to deliver its RSS 8-tuple.

At each reference point, a variable number of RSS measurements from each anchor node have been gathered to build a 8-tuple $\left\langle R S S_{\text {anchor 1 }} \ldots, R S S_{\text {anchor } 8}>\right.$ (these 8tuples are prepared to feed the LMS algorithm). Figure 8 represents the RSS measurements gathered from the 8th anchor node at the different 2D distances of 24 reference points. The curve is the fitting of eq. 1 to these experimental data. 


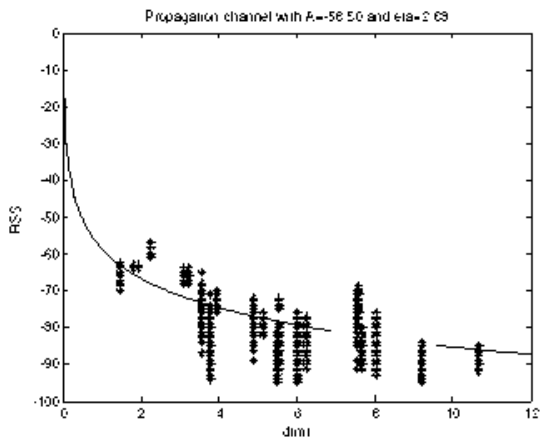

Figure 8. Propagation model with real data for the $8^{\text {th }}$ anchor node calculated on 24 reference points. RSS is measured in $\mathrm{dBm}$, and the propagation model paraneters are $A=-58.50 \mathrm{~dB}$ and $\eta=2.68$.

In a real scenario, an RSS 'measurement event' may be randonly generated in any of the available beacon points (depending on the people flow and activity), or may be generated in a periodic mode (if the reference point is conceived as part of the static infrastructure). For example, a user with a wireless equipped mobile device will deliver calibration measurements when passing on a reference point (the accurate detection of the user in the reference point may be done e.g. by using RFID-NFC landmarks). Additionally, infrastructure fixed reference points (e.g. a computer with a wireless adapter) will generate periodic tuples. So, with the obtained dataset, we have simulated the real process of calibration by feeding the LMS algorithm with tuples from randomly chosen reference points.

The performance of the algorithm is shown in Figure 9. under the same initial conditions that in the simulated scenario of the previous section, with $\mu_{\mathrm{A}}=0.1, \mu_{\mathrm{n}}=0.01$.

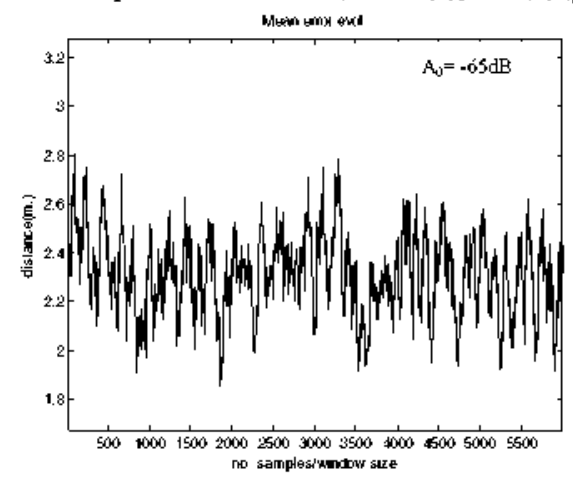

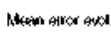

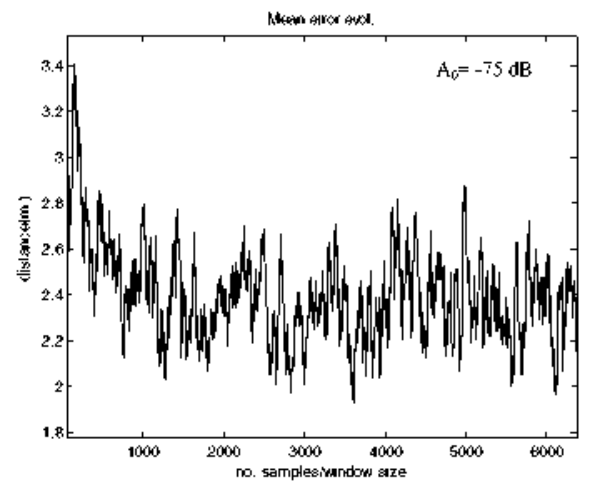

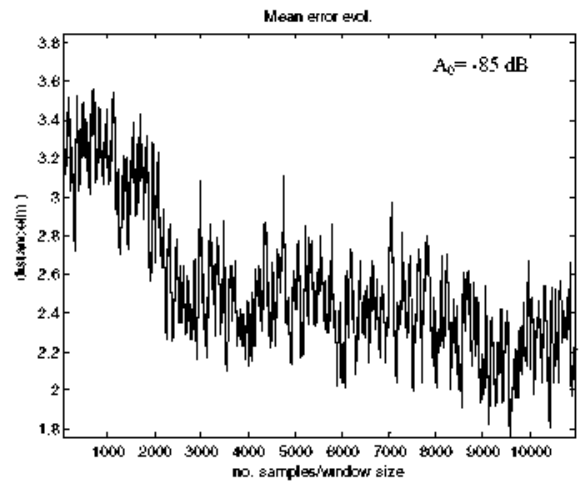

Figure 9. Evolution of the mean error ( $m$ ) in distance using a LMS filter with $\eta_{0}=3, \mu_{A}=0.1, \mu_{\eta}=0.01$ and different initial values for $\left.A_{0}: a\right)$ $65 \mathrm{~dB}, \mathrm{~b})-75 \mathrm{~dB}, \mathrm{c})-85$. The mean value is calculated with a sliding window of 50 samples over the arror in distance.

The mean error in distance shows a decreasing trend in every case in Figure 9; this behavior may be clearly identified for low values of $A$ and long-term training of the LMS algorithm. The number of samples needed to have a visible error reduction depends on the initial value of the LMS algorithm: 1300 samples when $A_{0}=-75 \mathrm{~dB}$, and 4000 samples when $A_{0}=-85 \mathrm{~dB}$ (approximately 166 samples per reference points are needed). For $A_{0}=-65 \mathrm{~dB}$, the error reduction is not so observable, although after processing approximately 800 samples, the average error is below 2.2 meters.

Figure 10 shows the real system's sensitivity to $A$ (by giving $\eta$ a fixed value). When $A$ is variable and $\eta$ set to 3 . the convergence value for $A$ is close to $-65 \mathrm{~dB}$. So. in the case in Figure 9, where $A_{4}=-65 \mathrm{~dB}$, the error reduction is not perceived as the best configuration for the propagation model coincides with the initial value given to $A$.

Apart from that, Figure 10 shows that $A$ can 'converge" with very different values of $\eta$, although with different stability and error reduction. As said before, $A$ is the dominant parameter in the lognormal propagation model (eq. 1), and it is affected by a step size one order of magnitude above the $\mu_{\eta}$.
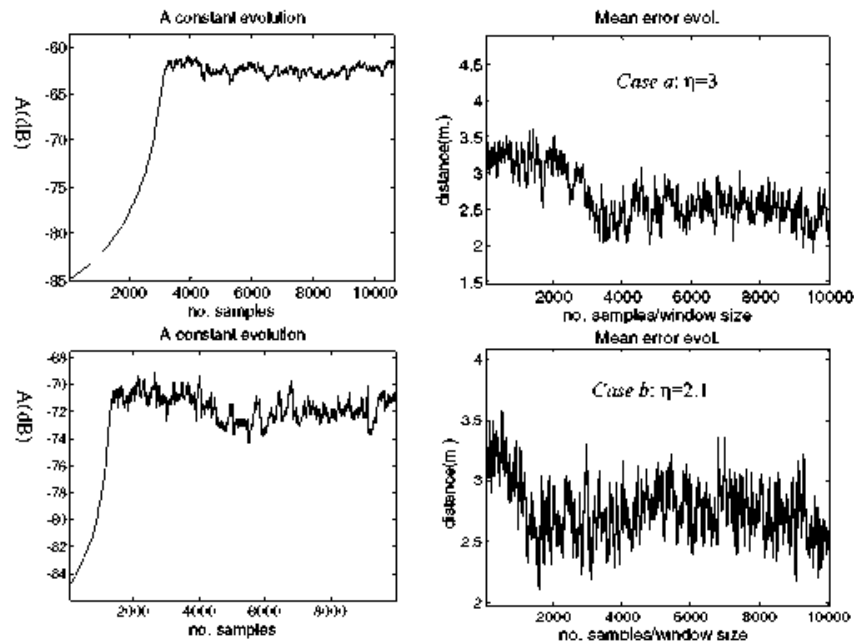

Figure 10. a) Evolution of $A$ and mean distance error when $\eta$ is invariable and set to a) 3, b) $2.1: A_{0}=-85 \mathrm{~dB}$. The LMS step sizes are $\mu_{A}=0.1, \mu_{\eta}=0.01$. 


\section{CONCLUSIONS AND FURTHER WORK}

Preliminary results in Section $\mathrm{V}$ and VI show that our LMS strategy for calibration is feasible and promising. The algorithm is capable to adapt the parameters of a lognomal propagation model to provide minimized errors in distance when using a hyperbolic localization technique.

The proposed algorithm is simple and lightweight in terms of computational needs and memory requirements: it performs iteratively on real-time data and the estimation obtained in the previous instant. For this reason, we find it especially attractive to assist localization systems in their calibration tasks, and also to embedded it in resource-constrained hardware (such as MicaZ motes) to perform distributed calibration.

In order to enhance the algorithm accuracy, we are currently testing the effect of handling various propagation models (one per anchor node. one per anchor node and room) and additional information about the physical stnicture of the environment (e.g. walls) to demonstrate if additional complexity in channel modeling may benefit the algorithm performance. Another idea we are working on is about sequencing the estimation of $\mathrm{A}$ and $\eta$. instead of doing joint computation. In the same line, we are also considering its integration with other localization algorithms (circular and weighted hyperbolic and circular).

From a practical viewpoint, an in-depth analysis about the relationship between the number of reference points, their geometrical distribution and the LMS algorithm accuracy is needed. It is also necessary to study the processing time the algorithm needs, in order to adjust the LMS step sizes to effectively work in real environments.

Together with the algorithm enhancement, it is necessary to explore how to easily deploy technologically-lybrid, unobtrusive and reconfigurable reference points in real environments, which guarantee the interaction with potential users to acquire sufficient calibration data in real-time operation.

As the reader will notice there is still a way to go to have a fully operative solution. In summary, further work is focused on demonstrating the stability of the proposal in real time operation. to show that the system can effectively adapt its behavior to real dynamic changes in the localization environment

\section{ACKNOWLEDGMENTS}

This work has been supported by the Government of Madrid under grant S-2009/TIC-1485 and by the Spanish Ministry of Science and Innovation under grant TIN200806742-C02-01. Authors acknowledge Henar Martín and Ines Ortega for their help with the empirical work.

\section{REFERENCES}

[1] Y. C. Chen. J. R. Chiang, H. H. Chu. P. Huang. A. W. Tsui "Sensor-assisted WiFi Indoor Location System for Adapting to Environmental Dynamics", Proc. of MSW LA, 2005.

[2] L. Chan. J, Chiang. Y. Chen. C. Ke. J. Hsu. H. Chu. Estimation with Neighborhood Links in Clusters", Proc. of the Internationat Conference on Pencasme Computing. pp. 50-66, 2006.

[3] A. W. Tsui, Y-H. Chuang. H-H. Chu, "Unsupervised Learning for Solving RSS Hardware Variance Problem in WiFi Localization", Mobile Netw. Appl, 14:667-691, 2009.

[4] H. Lui, H. Darabi. P. Banerjec. J. Liu, "Survey of Wireless Indoor Positioning Techniques and Systems", IEEE Trats. on Systems, Man and Cybernetics - Part C: Apphcations and Renews, vol. 37, no. 6, Novenuber 2007 .

[5] Y. Guon, R. Jain, "Error Characteristics and Calibration-fre Techniques for Wireless LAN-based Location Estimation". Procs. Of the Second International Workshop on Mobility Matagement \& Wreless Access Protocols (pp. 2-9), ACM. 2004.

[6] P. Barsocchi, S. Lenzi, S. Chessa, G. Giunta, "A Novel Approach to Indoor RSSI Localization by Automatic Calibration of the Wireless Propagation Model", Procs. of the $69^{\text {th }}$ Conf. on Vehcular Technology Conference, pp. 1-5, 2009.

[7] H. Lin, L-C. Kung. J.C. Hou. H. Luo, "Zero-configuration. robust indoor localization: Theory and Experinentation", Proc. IEEE INFOCOM $200625 T H$ IEEE International Conference on Computer Communications, pp. 1-12. IEEE Computer Society. 2006 .

[8] P. Krishnan. A.S. Krishnakumar, W-H. Ju. C. Mallows, S. Ganu. "A system for LEASE: Location Estimation Assisted by Stationary Enitters for Indoor RF Wireless Networks", Procs, of INFOCOM, pp. 1001 - 1011, vol.2, 2004

[9] L.F.M. de Moraes, B.A.A. Nunes, "Calibration-Free WLAN Location System Based on Dynamic Mapping of Signal Strength". Procs. of the 4th ACM international workshop on Mobility management and wireless access, pp. $92-99,2006$.

[10] J. Yin. Q. Yang, L. Ni. "Adaptive Temporal Radio Maps for Indoor Location Estimation". Procs. of the $3^{\text {th }}$ IEEE Int. Conf. on Pervasive Computing and Communications. 2005.

[11] V. W. Zheng. J. Zhao, Y. Wang, Q. Yang, "HIPS: A Calibrationless Hybrid Indoor Positioning System Using Heterogeneous Sensors", Procs. of the IEEE Int. Conf. on Pervasive Computing and Communications, 2009.

[12] L. M. Ni. Y. Lju, Y, C. Lau, A. P. Patil. "LANMARC: Indoor Location Sensing using Active RFID,"Wireless Networks, vol. 10, no. 6, pp. 701-710. Nov. 2004.

[13] P. Tarrío, A.M. Bernardos, J.R. Casar. "An RSS Localization Method based on Parametric Channel Models", Proceedings of the International Conference on Sensor Technologies and Applications, pp. 265-270, IEEE Computer Society, 2007.

[14] B. Widrow, J.M. McCool, M.G. Larimore, C.R. Jr. Johnson. "Stationary and nonstationary learning characteristics of the LMS adaptive filter," Proc. IEEE: vol, 64:pp. 1151-1162, Aug. 1976.

[15] P. Tartio, H. Martín, A.M. Bernardos, "Enhancing the Performance of Propagation Model-Based Positioning Algorithms". Procs. of the $3^{\text {rd }}$ International Workshop on UserCentric Technologies and Applications, pp. 123-132, 2009.

[16] MicaZ motes: http:i/www mensic.com 